PROCEEDINGS OF THE

AMERICAN MATHEMATICAL SOCIETY

Volume 130, Number 11, Pages 3269-3281

S 0002-9939(02)06610-8

Article electronically published on June 11, 2002

\title{
INTERPOLATION IN SELF-ADJOINT SETTINGS
}

\author{
Y. S. JO, J. H. KANG, R. L. MOORE, AND T. T. TRENT
}

(Communicated by David R. Larson)

\begin{abstract}
We study the operator equation $A X=Y$, where the operators $X$ and $Y$ are given and the operator $A$ is required to lie in some von Neumann algebra. We derive a necessary and sufficient condition for the existence of a solution $A$. The condition is that there must exist a constant $K$ so that, for all finite collections of operators $\left\{D_{1}, D_{2}, \ldots, D_{n}\right\}$ in the commutant, and all collections of vectors $\left\{f_{1}, f_{2}, \ldots, f_{n}\right\}$, we have $\left\|\sum_{j=1}^{n} D_{j} Y f_{j}\right\| \leq K\left\|\sum_{j=1}^{n} D_{j} X f_{j}\right\|$. We also study the equality $\|D Y f\|=K\|D X f\|$, in connection with solving the equation $A X=Y$ where the operator $A$ is required to lie in some CSL algebra.
\end{abstract}

Suppose that we are given a Hilbert space $\mathcal{H}$ and a weakly closed algebra $\mathcal{A}$ of operators acting on $\mathcal{H}$. Suppose also that two operators $X$ and $Y$ are specified, not necessarily in the algebra. Under what conditions can we expect there to be a solution of the operator equation $A X=Y$, where the operator $A$ is required to lie in $\mathcal{A}$ ? We refer to such a question as an interpolation problem. One might expect that the answer, if it can be found, would be highly dependent upon the nature of the algebra involved; for example, one might suppose that a singly generated algebra would require a different sort of answer from a CSL algebra. In fact, there are necessary conditions (not sufficient!) which can be specified universally. First, we fix some definitions and notation. Let $\mathcal{A}$ be a weakly closed algebra of operators on $\mathcal{H}$. We refer to the collection of subspaces that are invariant under all operators in $\mathcal{A}$ as the lattice of $\mathcal{A}$, denoted Lat $\mathcal{A}$. Actually, we like to ignore the distinction between a subspace and the orthogonal projection onto that subspace; it is often convenient to think of the lattice of an algebra as consisting of projections instead of subspaces.

Dually, suppose that $\mathcal{L}$ is a lattice of projections, containing 0 and the identity operator, and closed in the strong operator topology. (We always choose the lattice operations to be defined spatially; the join of two projections is the projection onto the span of their ranges, and the meet is the projection onto the intersection.) We denote by $\operatorname{Alg} \mathcal{L}$ the algebra of operators leaving invariant all of the projections in $\mathcal{L}$; this algebra contains the scalar multiples of the identity and is weakly closed. If an algebra $\mathcal{A}$ has the property that $\mathcal{A}=\operatorname{Alg}(\operatorname{Lat} \mathcal{A})$, then we say that $\mathcal{A}$ is reflexive. If a lattice $\mathcal{L}$ satisfies the equation $\mathcal{L}=\operatorname{Lat}(A \lg \mathcal{L})$, then $\mathcal{L}$ is also called reflexive. Every commutative lattice is reflexive 1 .

Now, suppose that a subalgebra $\mathcal{A}$ of $B(\mathcal{H})$ is given, along with two operators $X$ and $Y$, which do not necessarily lie in the algebra, and further suppose that there is an operator $A \in \mathcal{A}$ such that $A X=Y$. Let $E$ be a projection in Lat $\mathcal{A}$. For any

Received by the editors September 1, 2000 and, in revised form, June 7, 2001.

2000 Mathematics Subject Classification. Primary 46L10, 47L35. 
operator in the algebra, we then have $E^{\perp} A E^{\perp}=E^{\perp} A$. Thus, for any vector $f$,

$$
\begin{aligned}
A X f & =Y f, \\
E^{\perp} A X f & =E^{\perp} Y f, \\
E^{\perp} A E^{\perp} X f & =E^{\perp} Y f,
\end{aligned}
$$

and, consequently, we have

$$
\sup \left\{\frac{\left\|E^{\perp} Y f\right\|}{\left\|E^{\perp} X f\right\|}: f \in \mathcal{H} \text { and } E \in \mathcal{L}\right\} \leq\|A\|
$$

For these purposes, if $\left\|E^{\perp} X f\right\|=0$, then so does $\left\|E^{\perp} Y f\right\|$, so we can agree to ignore fractions of the form $0 / 0$ in computing the supremum above; equivalently, we can simply define $0 / 0$ to be 0 and these fractions will not affect the supremum. We will refer to the supremum above as $\rho(X, Y ; \mathcal{L})$, where $\mathcal{L}$ represents the lattice of the algebra $\mathcal{A}$. Thus, a necessary condition for the existence of an interpolating operator $A$ is that

$$
\rho(X, Y ; \mathcal{L})<\infty .
$$

The history of interpolation problems in operator algebras goes back at least to 1969, when Lance [5] studied the Jacobson radical of nest algebras. A nest of projections is a collection of subspaces that is linearly ordered by inclusion, and the corresponding nest algebra is the collection of all operators that leave invariant all the projections in the nest. Lance found it necessary to solve the operator equation $A x=y$, where $x$ and $y$ are given vectors, and $A$ is required to lie in some fixed nest algebra. This problem is equivalent to the version we have cited, if we take the operators $X$ and $Y$ to be rank-one with identical kernels and ranges $x$ and $y$ respectively. In Lance's case, the necessary condition $\rho(X, Y ; \mathcal{L})<\infty$ is also sufficient for the existence of a solution $A$. Hopenwasser [3] extended Lance's result to the case where the lattice of projections is commutative (a CSL) but may not be linearly ordered; in Hopenwasser's paper, the operators $X$ and $Y$ are still rank-one (although the problem there is stated in terms of single vectors rather than the equivalent version using rank-one operators). In this case, the necessary condition (A) is still sufficient. In [7], two of the authors of this paper took a different direction. Instead of restricting the operators $X$ and $Y$ to be rank-one, we allowed $X$ and $Y$ to be free, but required the lattice to be a nest and the algebra to be the corresponding nest algebra. Again, we found that the necessary condition $\rho(X, Y ; \mathcal{L})<\infty$ is sufficient for the existence of a solution to the equation $A X=Y$. However, we also noted in [7] that this condition may not always be sufficient for other lattices, even commutative ones.

\section{Part I. Von Neumann Algebras}

In this paper, we begin by considering the situation for von Neumann algebras. Von Neumann algebras have many invariant subspaces (all the projections in the commutant); however, the commutant may not be abelian, so our earlier results do not apply. We always consider von Neumann algebras to be concrete; that is, we work on a fixed Hilbert space $\mathcal{H}$, and our von Neumann algebras will be weakly closed, self-adjoint subalgebras of $B(\mathcal{H})$ (containing the identity operator). Let $\mathcal{V}$ be a von Neumann algebra and observe that, since $\mathcal{V}$ is self-adjoint, the lattice of $\mathcal{V}$ consists exactly of those projections that commute with $\mathcal{V}$; and, furthermore, $\mathcal{V}$ is 
the algebra of all operators which leave invariant all the projections that commute with $\mathcal{V}$. Thus, any von Neumann algebra is a reflexive algebra. The commutant of a von Neumann algebra $\mathcal{V}$ is the collection of all operators that commute with every operator in $\mathcal{V}$; the commutant is another von Neumann algebra, and the most important fact about the commutant is von Neumann's double commutant theorem: $\left(\mathcal{V}^{\prime}\right)^{\prime}=\mathcal{V}$. The projections in $\mathcal{V}^{\prime}$ determine $\mathcal{V}^{\prime}$. In the case of von Neumann algebras, it is easy to extend the necessary condition mentioned above.

Suppose that $\mathcal{V}$ is a von Neumann algebra and that $X$ and $Y$ are operators (not necessarily in $\mathcal{V})$. Suppose also that there is an operator $A \in \mathcal{V}$ such that $A X=Y$. For any $D \in \mathcal{V}^{\prime}$, we have

$$
D A X=D Y
$$

and so

$$
A D X=D Y
$$

and, for any $f \in \mathcal{H}$,

$$
A D X f=D Y f
$$

Consequently, we have the condition

$$
\sup \left\{\frac{\|D Y f\|}{\|D X f\|}: f \in \mathcal{H} \text { and } D \in \mathcal{V}^{\prime}\right\}<\infty .
$$

We can go further. If $\left\{D_{1}, D_{2}, \ldots, D_{n}\right\}$ are operators in $\mathcal{V}^{\prime}$ and $\left\{f_{1}, f_{2}, \ldots, f_{n}\right\}$ are arbitrary vectors, then

$$
A \sum_{j=1}^{n} D_{j} X f_{j}=\sum_{j=1}^{n} D_{j} A X f_{j}=\sum_{j=1}^{n} D_{j} Y f_{j}
$$

and consequently

$$
\left\|\sum_{j=1}^{n} D_{j} Y f_{j}\right\| \leq\|A\|\left\|\sum_{j=1}^{n} D_{j} X f_{j}\right\| .
$$

We thus have a necessary condition for interpolation via a von Neumann algebra, which is ostensibly stronger than condition (A) mentioned earlier. Appearances do not deceive: We will show later that condition (VN) is stronger than condition (A); first, we want to point our that it is sufficient for interpolation. The following theorem is a special case of a result that was first proved by Elias Katsoulis in [4]; actually, the proof of the special case and the more general theorem are identical, except for minor modifications, so we will omit the proof here and refer the reader to [4] for the details.

Theorem 1. Let $\mathcal{V}$ be a von Neumann algebra in $B(\mathcal{H})$, and let $X$ and $Y$ lie in $B(\mathcal{H})$. The following are equivalent:

(1) There exists an operator $A$ in $\mathcal{V}$ such that $A X=Y$.

(2) There is a constant $K$ such that, for any finite collections $\left\{D_{1}, D_{2}, \ldots, D_{n}\right\}$ of operators in $\mathcal{V}^{\prime}$ and vectors $\left\{f_{1}, f_{2}, \ldots, f_{n}\right\}$ in $\mathcal{H}$,

$$
\left\|\sum_{j=1}^{n} D_{j} Y f_{j}\right\| \leq K\left\|\sum_{j=1}^{n} D_{j} X f_{j}\right\|
$$

Furthermore, if condition (2) holds, then the operator mentioned in condition (1) can be chosen to have norm no larger than $K$. 
How does this condition compare to the general necessary condition (A), namely, that $\rho(X, Y ; \mathcal{L})<\infty$ ? Suppose that $\rho(X, Y ; \mathcal{L})=K$; then we know that, for any invariant projection $E$, and any vector $f$, we have $\left\|E^{\perp} Y f\right\| \leq K\left\|E^{\perp} X f\right\|$. This is equivalent to saying that $Y^{*} E^{\perp} Y \leq K^{2} X^{*} E^{\perp} X$. For von Neumann algebras, the lattice is complemented, so we can simply forget the " $\perp$ " symbol and write $Y^{*} E Y \leq K^{2} X^{*} E X$ for all projections $E \in \mathcal{V}^{\prime}$. Of course, if such a number $K$ exists, we can always normalize - for instance, by absorbing $K$ into $X$ - and assume that $K=1$.

Theorem 2. Let $\mathcal{V}$ be a von Neumann algebra in $B(\mathcal{H})$, and let $X$ and $Y$ lie in $B(\mathcal{H})$. The following are equivalent:

(1) For every projection $E$ in $\mathcal{V}^{\prime}$, and every vector $f \in \mathcal{H},\left\|E^{\perp} Y f\right\| \leq\left\|E^{\perp} X f\right\|$.

(2) For every operator $D \in \mathcal{V}^{\prime}$, and every vector $f \in \mathcal{H},\|D Y f\| \leq\|D X f\|$.

Proof. It is clear that (2) implies (1). Suppose that (1) holds; we will work with the version $Y^{*} E Y \leq X^{*} E X$. Suppose that $P$ is a positive operator in $\mathcal{V}^{\prime}$; then $P$ can be written as a limit of finite sums of the form $\sum \alpha_{j} E_{j}$, where, for each $j$, $E_{j}$ is a projection in $\mathcal{V}^{\prime}$ and $\alpha_{j}>0$. Consequently, $Y^{*} P Y \leq X^{*} P X$. Statement (2) now follows because it is equivalent to saying that $Y^{*} D^{*} D Y \leq X^{*} D^{*} D X$, and $D^{*} D \in \mathcal{V}^{\prime}$.

Thus, for von Neumann algebras, condition (A) and condition (VN1) are equivalent. However, these conditions involve just a single term, not a sum as would be needed for condition (VN). Unfortunately, we may not be able to infer the condition for sums from the condition for one term.

Example. Let $\mathcal{H}$ be three-dimensional complex space and let $\mathcal{K}$ represent $\mathcal{H} \oplus \mathcal{H}$. Then operators on $\mathcal{K}$ may be represented as $6 \times 6$ matrices, but we will choose to write them as $2 \times 2$ matrices with operator entries; the entries act on $\mathcal{H}$. Let $\mathcal{V}$ be the multiplicity- 2 von Neumann algebra

$$
\mathcal{V}=\{A \oplus A: A \in B(\mathcal{H})\}
$$

Operators in $\mathcal{V}$ can be written matricially as

$$
\left[\begin{array}{cc}
A & 0 \\
0 & A
\end{array}\right]
$$

and operators in the commutant have the form

$$
\left[\begin{array}{ll}
\alpha I & \beta I \\
\gamma I & \delta I
\end{array}\right]
$$

where the Greek letters can be any complex numbers and the identity $I$ acts on $\mathcal{H}$.

In this setting, suppose that $X$ and $Y$ are given matricially by

$$
X=\left[\begin{array}{ll}
X_{1} & 0 \\
X_{2} & 0
\end{array}\right] \quad \text { and } \quad Y=\left[\begin{array}{ll}
Y_{1} & 0 \\
Y_{2} & 0
\end{array}\right] .
$$

The matrix inequality $\|D X f\| \geq\|D Y f\|$, with $D$ in the commutant, is the same as the vector inequality

$$
\left\|\left[\begin{array}{c}
\left(\alpha X_{1}+\beta X_{2}\right) f \\
\left(\gamma X_{1}+\delta X_{2}\right) f
\end{array}\right]\right\| \geq\left\|\left[\begin{array}{c}
\left(\alpha Y_{1}+\beta Y_{2}\right) f \\
\left(\gamma Y_{1}+\delta Y_{2}\right) f
\end{array}\right]\right\|,
$$


which, since the complex numbers are arbitrary, amounts to the same as the inequality

$$
\left\|\left(\alpha X_{1}+\beta X_{2}\right) f\right\| \geq\left\|\left(\alpha Y_{1}+\beta Y_{2}\right) f\right\|,
$$

holding for all complex numbers $\alpha$ and $\beta$ and all vectors $f \in \mathcal{H}$.

Now let

$$
\begin{array}{ccc}
X_{1}=\left[\begin{array}{lll}
2 & 0 & 0 \\
0 & 0 & 0 \\
0 & 2 & 0
\end{array}\right], & X_{2}=\left[\begin{array}{lll}
0 & 0 & 0 \\
0 & 2 & 0 \\
2 & 0 & 0
\end{array}\right], \\
Y_{1}=\left[\begin{array}{lll}
1 & 0 & 0 \\
0 & 0 & 0 \\
0 & 2 & 0
\end{array}\right], & Y_{2}=\left[\begin{array}{lll}
0 & 0 & 0 \\
0 & 1 & 0 \\
1 & 0 & 0
\end{array}\right] .
\end{array}
$$

If we let $X_{\alpha \beta}$ represent the combination $\alpha X_{1}+\beta X_{2}$, and $Y_{\alpha \beta}$ similarly, then it is easy to compute that $X_{\alpha \beta}^{*} X_{\alpha \beta}-Y_{\alpha \beta}^{*} Y_{\alpha \beta}$ is the direct sum of the zero operator on one-dimensional space and the $2 \times 2$ matrix

$$
\left[\begin{array}{cc}
3\left(|\alpha|^{2}+|\beta|^{2}\right) & 2 \alpha \bar{\beta} \\
2 \alpha \bar{\beta} & 3|\beta|^{2}
\end{array}\right]
$$

which is easily seen to be a positive matrix. Consequently, we have that $\left\|X_{\alpha \beta} f\right\| \geq$ $\left\|Y_{\alpha \beta} f\right\|$ for all $\alpha, \beta$, and $f \in \mathcal{H}$; so $\|D X f\| \geq\|D Y f\|$ for all $D \in \mathcal{V}^{\prime}$, as desired. On the other hand, in order to have an operator in $\mathcal{V}$ that maps $X$ to $Y$, we would need an operator $A$ acting on $\mathcal{H}$ so that $A X_{1}=Y_{1}$ and $A X_{2}=Y_{2}$. It is quite easy to check that there is simply no such matrix, even with large norm; in particular, the $(3,3)$-entry of such a matrix would have to satisfy the two equations $x=1$ and $2 x=1$.

Question. Can a similar counterexample be constructed, but with $\mathcal{H}$ two-dimensional instead of three-dimensional? It would be desirable to have as simple an example as possible.

It is clear that the multiplicity of the von Neumann algebra plays an important role in this example. If there were no multiplicity involved, we would only need two operators $A$ and $B$ so that $A X_{1}=Y_{1}$ and $B X_{2}=Y_{2}$, instead of a single operator that does both jobs; that would be easy. In fact, if multiplicity is not present, we only need a single term in condition (VN).

Theorem 3. Suppose that $\mathcal{V}$ is a multiplicity-free von Neumann algebra, and suppose that $\|D X f\| \geq\|D Y f\|$ for all $D \in \mathcal{V}^{\prime}$ and all $f \in \mathcal{H}$. Then there exists an operator $A$ in $\mathcal{V}$ such that $A X=Y$ and $\|A\| \leq 1$.

Proof (sketch). Use the fact that a multiplicity-free von Neumann algebra can be written as the direct integral of full matrix algebras to reduce to the case that $\mathcal{V}=B(\mathcal{H})$. In this case, the well-known range inclusion theorem of R. G. Douglas [2] provides the result.

Whether there is multiplicity or not, if $X$ and $Y$ are in the algebra themselves, life is simpler. Notice that the commutant of the von Neumann algebra doesn't even come into the hypothesis of the next theorem. 
Theorem 4. Let $\mathcal{V}$ be a von Neumann subalgebra of $B(\mathcal{H})$ and let $X$ and $Y$ lie in $\mathcal{V}$. Suppose that, for all $f \in \mathcal{H}$, we have $\|X f\| \geq\|Y f\|$. Then there exists an operator $A \in \mathcal{V}$ such that $A X=Y$ and $\|A\| \leq 1$.

Proof. With the condition given, suppose that $D_{j} \in \mathcal{V}^{\prime}$. Then, for any $f_{j}$,

$$
\begin{aligned}
\left\|\sum D_{j} X f_{j}\right\| & =\left\|\sum X D_{j} f_{j}\right\| \\
& =\left\|X\left(\sum D_{j} f_{j}\right)\right\| \\
& \geq\left\|Y\left(\sum D_{j} f_{j}\right)\right\| \\
& =\left\|\sum D_{j} Y f_{j}\right\|
\end{aligned}
$$

which is condition $(\mathrm{VN})$.

To get a better grip on how the conditions (VN) and (A) compare, we want to look at inflations of the von Neumann algebra; in other words, we need to introduce multiplicity, even if none were present at the outset.

Let $\mathcal{V}$ be a von Neumann algebra of operators acting on a Hilbert space $\mathcal{H}$ and let $\mathcal{M}_{n}$ represent the collection of $n \times n$ matrices, with identity operator $I_{n}$. We denote by $\mathcal{H}^{(n)}$ the direct sum of $n$ copies of $\mathcal{H}: \mathcal{H}^{(n)}=\mathcal{H} \oplus \mathcal{H} \oplus \mathcal{H} \oplus \ldots \oplus \mathcal{H}$. The direct sum of Hilbert spaces may also be thought of as $\mathcal{H} \otimes \mathbb{C}^{n}$, where we think of the tensor product of Hilbert spaces as consisting of vectors of the form $\sum f_{j} \otimes \xi_{j}$, where $f_{j} \in \mathcal{H}$ and $\xi_{j} \in \mathbb{C}^{n}$. We define

$$
\mathcal{V} \otimes I_{n}=\{A \oplus A \oplus A \oplus \ldots \oplus A: A \in \mathcal{V}\} .
$$

Then it is easy to see that $\mathcal{V} \otimes I_{n}$ is a von Neumann algebra. What is its commutant? For $D \in B(\mathcal{H})$, and $S \in \mathcal{M}_{n}$, we define $(D \otimes S)(f \otimes \xi)=D f \otimes S \xi$, extended to be linear. Then $D \otimes S$ acts on $\mathcal{H} \otimes \mathbb{C}^{n}$, and has norm equal to $\|D\|\|S\|$. One can realize $D \otimes S$ matricially as follows. Suppose that $S=\left[s_{i j}\right]_{i, j=1 \ldots n}$. Then $D \otimes S=\left[s_{i j} D\right]_{i, j=1 \ldots n}$, acting on $\mathcal{H}^{(n)}$. If we restrict the operator $D$ to lie in $\mathcal{V}^{\prime}$, then sums of operators of the form $D_{j} \otimes S_{j}$ form the von Neumann algebra denoted by $\mathcal{V}^{\prime} \otimes \mathcal{M}_{n}$. It is easy to check that any such operator lies in the commutant of $\mathcal{V} \otimes I_{n}$, and, indeed, the commutant consists of the closure of the set of sums of elementary tensors; this fact is an easy consequence of the celebrated TomitaTakesaki commutant theorem, but it isn't hard to give a self-contained proof in this easy case.

For simplicity, we refer to $A \oplus A \oplus \ldots \oplus A$ as $A^{(n)}$. Suppose now that $A X=Y$. Then $A^{(n)} X^{(n)}=Y^{(n)}$ and clearly $\left\|A^{(n)}\right\|=\|A\|$. Now $\mathcal{V} \otimes I_{n}$ is another von Neumann algebra, with commutant $\mathcal{V}^{\prime} \otimes \mathcal{M}_{n}$, so we can invoke condition (VN1) for the equation $A^{(n)} X^{(n)}=Y^{(n)}$. For instance, if $n=2$, we have

$$
\left[\begin{array}{cc}
D_{1} & D_{2} \\
D_{3} & D_{4}
\end{array}\right]\left[\begin{array}{cc}
X & 0 \\
0 & X
\end{array}\right]\left[\begin{array}{l}
f_{1} \\
f_{2}
\end{array}\right]=\left[\begin{array}{l}
D_{1} X f_{1}+D_{2} X f_{2} \\
D_{3} X f_{1}+D_{4} X f_{2}
\end{array}\right]
$$

where each $D_{j}$ is in $\mathcal{V}^{\prime}$. To say that $\left\|D X^{(2)} f\right\| \geq\left\|D Y^{(2)} f\right\|$ for all $D \in\left(\mathcal{V} \otimes I_{2}\right)^{\prime}$ and all $f \in \mathcal{H} \oplus \mathcal{H}$ is equivalent, as in the example, to the single inequality

$$
\left\|D_{1} X f_{1}+D_{2} X f_{2}\right\| \geq\left\|D_{1} Y f_{1}+D_{2} Y f_{2}\right\|
$$


for all $D_{1}, D_{2}$ in $\mathcal{V}^{\prime}$ and all $f_{1}, f_{2} \in \mathcal{H}$. By extension we have

Theorem 5. Let $\mathcal{V}$ be a von Neumann subalgebra of $B(\mathcal{H})$, and let $X, Y$ lie in $B(\mathcal{H})$. Let $n$ be a positive integer. The following are equivalent:

(i) For all $n$-tuples of operators $\left\{D_{1}, D_{2}, \ldots, D_{n}\right\}$ in $\mathcal{V}^{\prime}$ and vectors $\left\{f_{1}, f_{2}, \ldots\right.$, $\left.f_{n}\right\}$ in $\mathcal{H}$, we have

$$
\left\|\sum_{j=1}^{n} D_{j} X f_{j}\right\| \geq\left\|\sum_{j=1}^{n} D_{j} Y f_{j}\right\| .
$$

(ii) For all $D \in\left(\mathcal{V} \otimes I_{n}\right)^{\prime}$, and $f \in \mathcal{H}^{(n)}$, we have

$$
\left\|D X^{(n)} f\right\| \geq\left\|D Y^{(n)} f\right\| .
$$

Consequently, we see that, if and only if condition (ii) holds for all $n$, there is an interpolating operator $A \in \mathcal{V}$, such that $A X=Y$ and $\|A\| \leq 1$. In the example above, it is not hard to show that, even though the condition holds for $n=1$, it fails for $n=2$.

We would like to have a systematic way of constructing such examples. To be more specific, we adopt the following notation:

$$
\sigma(X, Y ; \mathcal{V})=\sup \left\{\frac{\|D Y f\|}{\|D X f\|}: f \in \mathcal{H} \text { and } D \in \mathcal{V}^{\prime}\right\} .
$$

We also let $\sigma_{n}(X, Y ; \mathcal{V})=\sigma\left(X^{(n)}, Y^{(n)} ; \mathcal{V}^{(n)}\right\}$. It is easy to see that the sequence $\left\{\sigma_{n}(X, Y ; \mathcal{V})\right\}$ is non-decreasing as $n$ increases. Furthermore, if $A \in \mathcal{V}$ and $A X=Y$, then $\|A\| \geq \sup \left\{\sigma_{n}(X, Y ; \mathcal{V}): n \in \mathbb{N}\right\}$, and, from what we have just proved, if the supremum is finite, then there exists an interpolating operator whose norm is equal to the supremum.

Question. For each $n$, find an example so that $\sigma_{n}(X, Y ; \mathcal{V})$ is strictly less than $\sigma_{n+1}(X, Y ; \mathcal{V})$, and so that $\sigma_{n+1}(X, Y ; \mathcal{V})=\sup \left\{\sigma_{k}(X, Y ; \mathcal{V}): k=1, \ldots, \infty\right\}$.

Question. If the operators in question act on a $k$-dimensional space, is it true that $\sigma_{k}(X, Y ; \mathcal{V})=\sup \left\{\sigma_{n}(X, Y ; \mathcal{V}): n \in \mathbb{N}\right\}$. Is there a better estimate? For instance, in our example the operators act on 6 -dimensional space but $\sigma_{2}$ tells the whole story.

\section{PART II. RePlacing INEQUALities By EQUATIONS}

Let $\mathcal{A}$ be an algebra of operators, with lattice of invariant subspaces $\mathcal{L}=\operatorname{Lat} \mathcal{A}$. In the preamble, we observed that, if we hope to find an operator $A \in \mathcal{A}$ such that $A X=Y$, then the collection $\left\{\frac{\left\|E^{\perp} Y f\right\|}{\left\|E^{\perp} X f\right\|}: E \in \mathcal{L}\right\}$ must be bounded (with the convention $0 / 0=0$ ). If it is, then we can normalize and assume that the bound is 1, so that $\left\|E^{\perp} Y f\right\| \leq\left\|E^{\perp} X f\right\|$ for all $E \in \mathcal{L}$ and all $f \in \mathcal{H}$; equivalently, $Y^{*} E^{\perp} Y \leq X^{*} E^{\perp} X$ for all $E$ in $\mathcal{L}$. In this section we consider the much stronger condition

$$
Y^{*} E^{\perp} Y=X^{*} E^{\perp} X
$$

for all $E \in \mathcal{L}$, with $\mathcal{L}$ commutative. In this case, we can prove a much stronger result. Furthermore, we do not need to assume anything about inflations; we get them for free. 
Theorem 6. Let $\mathcal{L}$ be a commutative subspace lattice acting on the Hilbert space $\mathcal{H}$, and let $\mathcal{A}=A \operatorname{Alg} \mathcal{L}$. Let $X$ and $Y$ be any two operators in $B(\mathcal{H})$. The following are equivalent:

(1) $Y^{*} E^{\perp} Y=X^{*} E^{\perp} X$, for all $E \in \mathcal{L}$.

(2) There is a partial isometry $V$ in the diagonal of $\mathcal{A}$ such that $V X=Y$ and $V^{*} Y=X$.

In order to demonstrate this result, we will use the main theorem in the previous section. Actually, we want a refinement of that theorem.

Theorem 7. Let $\mathcal{H}$ be a Hilbert space, and let $\mathcal{V}$ be a von Neumann subalgebra of $B(\mathcal{H})$ with abelian commutant. Suppose that $X$ and $Y$ are operators on $\mathcal{H}$ and suppose that, for all projections $P \in \mathcal{V}^{\prime}$, and for all $f \in \mathcal{H}$, we have $\|P X f\|=$ $\|P Y f\|$. Then there is a partial isometry $V$ in $\mathcal{V}^{\prime}$ so that $V X=Y$ and $V^{*} Y=X$.

Proof. We hope to show eventually that, whenever $D_{1}, D_{2}, \ldots, D_{n}$ are operators in $\mathcal{V}^{\prime}$, and $f_{1}, f_{2}, \ldots, f_{n}$ are vectors in $\mathcal{H}$, then $\left\|\sum D_{i} X f_{i}\right\|=\left\|\sum D_{i} Y f_{i}\right\|$. First, suppose that $P_{1}, P_{2}, \ldots, P_{n}$ is a collection of mutually orthogonal projections in $\mathcal{V}^{\prime}$. Then we have

$$
\begin{aligned}
\left\|\sum P_{i} X f_{i}\right\|^{2} & =\sum\left\|P_{i} X f_{i}\right\|^{2} \\
& =\sum\left\|P_{i} Y f_{i}\right\|^{2} \\
& =\left\|\sum P_{i} Y f_{i}\right\|^{2} .
\end{aligned}
$$

Next, suppose that $P_{1}, P_{2}, \ldots, P_{n}$ is a collection of projections in $\mathcal{V}^{\prime}$, but that the projections are not necessarily mutually orthogonal. We break up the appropriate sum in the following lengthy way:

$$
\begin{aligned}
\sum_{i=1}^{n} P_{i} X f_{i} & =\left(P_{1} P_{2} \ldots P_{n}\right) X\left(f_{1}+f_{2}+\cdots+f_{n}\right) \\
& +\left(P_{1}^{\perp} P_{2} \ldots P_{n}\right) X\left(f_{2}+\cdots+f_{n}\right) \\
& +\left(P_{1} P_{2}^{\perp} P_{3} \ldots P_{n}\right) X\left(f_{1}+f_{3}+\cdots+f_{n}\right) \\
& +\ldots \\
& +\left(P_{1} P_{2} \ldots P_{n-1} P_{n}^{\perp}\right) X\left(f_{1}+\ldots f_{n-1}\right) \\
& +\left(P_{1}^{\perp} P_{2}^{\perp} P_{3} \ldots P_{n}\right) X\left(f_{3}+\cdots+f_{n}\right) \\
& +\left(P_{1}^{\perp} P_{2} P_{3}^{\perp} P_{4} \ldots P_{n}\right) X\left(f_{2}+f_{4}+\cdots+f_{n}\right) \\
& +\ldots \\
& +\left(P_{1}^{\perp} P_{2}^{\perp} \ldots P_{n-1}^{\perp} P_{n}\right) X f_{n} \\
& +\ldots \\
& +\left(P_{1} P_{2}^{\perp} \ldots P_{n}^{\perp}\right) X f_{1} .
\end{aligned}
$$

If we want to make the notation truly impenetrable, we can write it this way:

$$
\sum_{i=1}^{n} P_{i} X f_{i}=\sum_{S \subseteq\{1,2, \ldots, n\}}\left(\prod_{j \in S} P_{j}\right)\left(\prod_{j \notin S} P_{j}^{\perp}\right) X\left(\sum_{j \in S} f_{j}\right) .
$$

In either case, the point is that the sum on the right-hand side is now written so that any two terms are orthogonal. Consequently, the square of the norm is 
the sum of the squares of the norms of the terms on the right-hand side. On the other hand, every term has the form $P X f$, for some choice of projection $P \in \mathcal{V}^{\prime}$ and $f \in \mathcal{H}$. (Remember that $\mathcal{V}^{\prime}$ is abelian, so products of projections are just projections again.) But $\|P X f\|=\|P Y f\|$. Thus, upon replacing $X$ by $Y$ in each term, we do not change the norm. Upon recombining, we get the result

$$
\left\|\sum_{1}^{n} P_{i} X f_{i}\right\|=\left\|\sum_{1}^{n} P_{i} Y f_{i}\right\|
$$

Since scalar multiples $\left\{\lambda_{i}\right\}$ can be absorbed into the vectors if desired, we can write

$$
\left\|\sum_{1}^{n} \lambda_{i} P_{i} X f_{i}\right\|=\left\|\sum_{1}^{n} \lambda_{i} P_{i} Y f_{i}\right\|
$$

Now, suppose that $D_{1}, D_{2}, \ldots, D_{n}$ are any operators in $\mathcal{V}^{\prime}$. Since $\mathcal{V}^{\prime}$ is abelian, each $D_{j}$ is normal and we can use the spectral theorem to write $D_{j}$ as a strong limit of operators of the form $\sum_{1}^{m} \lambda_{k} P_{k}$, where each projection $P_{k}$ lies in $\mathcal{V}^{\prime}$. Therefore, the sum $\sum_{1}^{n} D_{i} X f_{i}$ is a (norm) limit of sums of the form

$$
\sum_{i=1}^{n} \sum_{k=1}^{m_{n}} \lambda_{k i} P_{k i} X f_{i}
$$

which is precisely of the form mentioned in $(*)$. As a result, we have the desired fact:

$$
\left\|\sum_{1}^{n} D_{i} X f_{i}\right\|=\left\|\sum_{1}^{n} D_{i} Y f_{i}\right\| .
$$

We are almost finished. We proceed now as in the previous section. Define an operator $V$ as follows:

$$
V: \sum_{1}^{n} D_{i} X f_{i} \longmapsto \sum_{1}^{n} D_{i} Y f_{i} .
$$

It is a special case of the last equation that $A X=Y$. As before, we define $V$ to be 0 on the orthogonal complement of the subspace spanned by elements of the form $\sum_{1}^{n} D_{i} X f_{i}$. It follows as before that $V$ lies in the von Neumann algebra $\mathcal{V}^{\prime}$. Furthermore, it is obvious from the definition that $V$ is a partial isometry, whose initial space is the span of vectors of the form $\sum D_{i} X f_{i}$; this space contains the range of $X$. Since $V X=Y$, we have $X=V^{*} V X=V^{*} Y$. The proof is complete.

Proof of Theorem 6. It is obvious that the existence of the partial isometry implies the equality of $\left\|Y^{*} E^{\perp} Y\right\|$ and $\left\|X^{*} E^{\perp} X\right\|$.

Now suppose that $\mathcal{L}$ is a commutative subspace lattice, and that $Y^{*} E^{\perp} Y=$ $X^{*} E^{\perp} X$ for all $E \in \mathcal{L}$. Since $E=0$ is one possibility, we have $Y^{*} Y=X^{*} X$. Now, by subtracting the equalities, we have $Y^{*} E Y=X^{*} E X$ for any $E \in \mathcal{L}$. A further subtraction now shows that $Y^{*}(E-F) Y=X^{*}(E-F) X$ whenever $E$ and $F$ lie in $\mathcal{L}$ with $F \leq E$. Now let $\mathcal{V}$ represent the diagonal of $A l g \mathcal{L}$, that is, $\mathcal{V}=A \lg \mathcal{L} \cap(A \lg \mathcal{L})^{*}$ and let $\mathcal{V}_{1}$ be the von Neumann algebra generated by all intervals $E-F$, with $E, F \in \mathcal{L}$. It is clear that $\mathcal{V}^{\prime}=\mathcal{V}_{1}$, and that $\mathcal{V}_{1}$ is abelian. Furthermore, the equality above shows that $Y^{*} P Y=X^{*} P X$ for all projections $P \in \mathcal{V}_{1}$. The desired result now follows from Theorem 7 . 


\section{Part III. Multivariable Toeplitz OPERAtors}

We want to apply the results of the previous sections to the factorization of Toeplitz operators. We are especially interested in Toeplitz operators acting in several complex variables.

To fix notation, let $D$ be the unit disk in the complex plane, let $C$ be a Hilbert space of dimension $N$ (where $N \leq \infty$ ) and let $H_{C}^{2}$ represent vector-valued Hardy space - that is, functions acting on the unit disk with values in the Hilbert space $C$. We denote by $S_{C}$ the operator given by multiplication by $z$ acting on $H_{C}^{2}$. Of course, $S_{C}$ is just a unilateral shift of multiplicity $N$; we denote by $S$ the usual unilateral shift (of multiplicity 1 ). There are two ways to view $H_{C}^{2}$; we can picture it as the direct $\operatorname{sum} \sum_{1}^{N} \oplus H^{2}(D)$ or as the tensor product $C \otimes H^{2}(D)$. Thus, the commutant of $S_{C}$ will have two natural representations. If $F \in\left\{S_{C}\right\}^{\prime}$, then we might view $F$ as an $N \times N$ operator matrix with analytic Toeplitz operators for entries. On the other hand, we can regard $F$ as an infinite operator matrix with operator entries $F_{i j} \in B(C)$ that satisfy the usual analytic Toeplitz criteria: (1) $F_{i j}=0$ if $j>i$; (2) $F_{i j}=F_{i+1, j+1}$ for all $i, j=0,1,2, \ldots$ Taking the first viewpoint, $S_{C}$ itself is an $N \times N$ diagonal matrix with diagonal entries all equal to $S$; in the second view, $S_{C}$ is a matrix whose entries on the first subdiagonal are all equal to $I_{C}$, with zeroes elsewhere. We remark that if $F$ and $F^{*}$ both commute with $S_{C}$, then we can view $F$ as either (1) an $N \times N$ operator matrix with entries that are constant multiples of $I_{C}$; or (2) a diagonal matrix with diagonal entries that are all equal to the same operator in $B(C)$.

Our first result is just a recasting of Theorem 6. Our main reason for working with the orthogonal complements $E_{n}^{\perp}$ and the adjoints $F^{*}$ and $G^{*}$ in the next theorem is that we prefer to view matrices as upper triangular instead of lower triangular.

Theorem 8. Let $F$ and $G$ lie in $\left\{S_{C}\right\}^{\prime}$ and suppose that $F F^{*}=G G^{*}$. Then there exists a partial isometry $V$ such that $V$ and $V^{*}$ both lie in $\left\{S_{C}\right\}^{\prime}$ and so that

$$
V F^{*}=G^{*} \quad \text { and } \quad F^{*}=V^{*} G^{*} .
$$

Proof. We will use the second representation for $\left\{S_{C}\right\}^{\prime}$. Let $E_{n}^{\perp}$ denote the projection of $H^{2}(D)$ onto $z^{n} H^{2}(D)$. Similarly, let $\mathcal{E}^{\perp}=I_{C} \otimes E_{n}^{\perp}$. Then $E_{n}^{\perp}=S^{n} S^{* n}$ and $\mathcal{E}^{\perp}=S_{C}^{n} S_{C}^{* n}$. Since $F$ and $G$ commute with $S_{E}$, we have

$$
\begin{aligned}
F \mathcal{E}^{\perp} F^{*} & =F S_{C}^{n} S_{C}^{* n} F^{*}=S_{C}^{n} F F^{*} S_{C}^{* n} \\
& =S_{C}^{n} G G^{*} S_{C}^{* n}=G S_{C}^{n} S_{C}^{* n} G^{*} \\
& =G \mathcal{E}^{\perp} G^{*} .
\end{aligned}
$$

We let $\mathcal{L}$ be the lattice generated by the projections $\mathcal{E}^{\perp}$; since finite meets and joins of the projections in $\mathcal{L}$ can be written as sums and products of the elementary tensors $\mathcal{E}^{\perp}$, we have

$$
F \mathcal{E} F^{*}=G \mathcal{E} G^{*}
$$

for all $\mathcal{E}$ in $\mathcal{L}$.

The algebra of the lattice $\mathcal{L}$ consists of upper triangular operator-valued matrices; the operator entries lie in $B(C)$. The algebra $\left\{S_{C}^{*}\right\}^{\prime}$ is a subset of this algebra. By Theorem 6, there is a partial isometry $U$ in the diagonal of the algebra $A l g \mathcal{L}$ such that $U F^{*}=G^{*}$ and $F^{*}=U^{*} G^{*}$. To show that we can choose an interpolating 
operator in the commutant, let $U=\operatorname{diag}\left(U_{0}, U_{1}, \ldots\right)$. Then $U F^{*}=G^{*}$ gives us that $U_{0} F_{i j}^{*}=G_{i j}^{*}$ for all $i, j$. Consequently, the operator $V=\operatorname{diag}\left(U_{0}, U_{0}, \ldots\right)$ will also satisfy the requirement that $V F^{*}=G^{*}$ and $V^{*} G^{*}=F^{*}$. Since $U$ is a partial isometry, so is $U_{0}$ and we are done.

For the next results, we require two lemmas. For the first of these, we need to view $S_{C}$ as a diagonal $N \times N$ matrix whose diagonal entries are all equal to the simple unilateral shift. In this case, if $T$ is an operator in the commutant of $S_{C}$, then we can write $T$ as an $N \times N$ operator matrix with entries that are all analytic Toeplitz operators: $T=\left[T_{f_{i j}}\right]_{i, j=1}^{N}$. Each of the functions $f_{i j}$ is an analytic function on the unit disk. Therefore, we can consider the associated matrices $T(z)$ defined by

$$
T(z)=\left[f_{i j}(z)\right]
$$

which are all $N \times N$ matrices with complex numerical entries.

Lemma 9. Let $T$ lie in $\left\{S_{C}\right\}^{\prime}$ and let $T$ be represented by the $N \times N$ matrix $\left[T_{f_{i j}}\right]_{i, j=1}^{N}$, where, for all $i$ and $j, f_{i j} \in H^{\infty}(D)$. Then the norm of $T$ is given by

$$
\|T\|=\sup _{z \in D}\|T(z)\|
$$

Proof. In order to distinguish vectors in $H^{2}(D)$ from those in $H_{C}^{2}(D)$, we will use underlined letters for the latter space. Fix a function $\underline{h}$ in $H_{C}^{2}(D)$, which is the space on which the operator $T$ acts. We write $\underline{h}$ as the $N$-vector $\underline{h}=\left[h_{j}\right]_{j=1}^{N}$. Then the vector $T \underline{h}$ is an $N$-vector whose components are given by $[T \underline{h}]_{i}=\sum_{j=1}^{N} f_{i j} h_{j}$. Using $\sigma$ for normalized Lebesgue measure on the unit circle, we have

$$
\begin{aligned}
\|T \underline{h}\|_{H_{C}^{2}(D)}^{2} & =\int_{\partial D}\|T(z) \underline{h}(z)\|^{2} d \sigma \\
& \leq \int_{\partial D}\|T(z)\|^{2}\|\underline{h}(z)\|_{C}^{2} d \sigma \\
& \leq\left(\sup _{z \in D}\|T(z)\|^{2}\right) \cdot \int_{\partial D}\|\underline{h}(z)\|^{2} d \sigma \\
& \leq\left(\sup _{z \in D}\|T(z)\|^{2}\right) \cdot\|\underline{h}\|_{H_{C}^{2}(D)}^{2}
\end{aligned}
$$

which establishes the inequality $\|T\| \leq \sup _{z \in D}\|T(z)\|$.

On the other hand, let $\xi$ be a vector in the space $C$, and let $k_{z}$ be the usual reproducing kernel for $H^{2}(D)$. Then, in a natural way, we can view $k_{z} \xi$ as a vector in $H_{C}^{2}(D)$. Moreover, because the reproducing kernel is an eigenvector of $T_{f}^{*}$, it is not hard to see that $T^{*} k_{z} \xi=k_{z} \cdot\left(T(z)^{*} \xi\right)$. We have

$$
\begin{aligned}
\left\|T^{*}\left(k_{z} \xi\right)\right\| & =\left\|\left[T_{f_{i j}}\right]^{*}\left(k_{z} \xi\right)\right\| \\
& =\left\|k_{z} T(z)^{*} \xi\right\| \\
& =\|T(z) \xi\|\left\|k_{z}\right\| \\
& =\|T(z) \xi\| .
\end{aligned}
$$

By choosing a unit vector $\xi$ to maximize the norm of the last line, we see that, for any $z,\|T\| \geq\|T(z)\|$, which completes the proof. 
The next lemma refers to reproducing kernel Hilbert spaces. We assume that the set $\Omega$ is a bounded, connected open subset of $\mathbb{C}^{n}$. A general reference for reproducing kernel Hilbert spaces is the book by Meschkowski [6].

Lemma 10. Let $H(\Omega)$ be a reproducing kernel Hilbert space of analytic functions on a region $\Omega \subseteq \mathbb{C}^{n}$, with reproducing kernel $k_{z}$. Suppose that $A \in B(H(\Omega))$ and suppose also that

$$
\left\langle A k_{z}, k_{z}\right\rangle=0 \quad \text { for all } z \text { in } \Omega
$$

Then $A=0$.

Proof. This result is due to Berezin. Note that the mapping

$$
z \longmapsto \frac{\left\langle A k_{z}, k_{z}\right\rangle}{\left\|k_{z}\right\|^{2}}
$$

is the Berezin symbol for $A$. One reference for the proof is Moore-Trent 8 .

Corollary 11. Let $\left\{f_{j}\right\}$ and $\left\{g_{j}\right\}$ be sequences of functions defined and analytic on a region $\Omega \subset \mathbb{C}$. Suppose that there is a constant $C<\infty$ so that

$$
\sum_{j=1}^{\infty}\left|f_{j}(z)\right|^{2}=\sum_{j=1}^{\infty}\left|g_{j}(z)\right|^{2} \leq C^{2}
$$

for all $z$ in some open disk $D$ contained in $\Omega$. Then there is a partial isometry $U$ in $B\left(\ell^{2}\right)$ so that $U \underline{F}(z)=\underline{G}(z)$ and $\underline{F}(z)=U^{*} \underline{G}(z)$ for all $z \in \Omega$, where $\underline{F}(z)=\left(f_{1}(z), f_{2}(z), \ldots\right)$, and likewise for $\underline{G}(z)$.

Proof. By an argument using connectedness and the permanence of functional relations, we will assume that $\Omega=D=$ the unit disk. By Lemma 9 we have

$$
\left\|\sum T_{f_{j}} T_{f_{j}}^{*}\right\|^{2}=\sup _{z \in D} \sum\left|f_{j}(z)\right|^{2} \leq C^{2}
$$

and likewise for $\left\|\sum T_{g_{j}} T_{g_{j}}^{*}\right\|^{2}$. For $z \in D$ we have

$$
\begin{aligned}
\left\langle\left(\sum T_{f_{j}} T_{f_{j}}^{*}\right) k_{z}, k_{z}\right\rangle & =\sum\left|f_{j}(z)\right|^{2}\left\langle k_{z}, k_{z}\right\rangle \\
& =\sum\left|g_{j}(z)\right|^{2}\left\langle k_{z}, k_{z}\right\rangle \\
& =\left\langle\left(\sum T_{g_{j}} T_{g_{j}}^{*}\right) k_{z}, k_{z}\right\rangle
\end{aligned}
$$

where $k_{z}$ is the reproducing kernel for $h^{2}(D)$. By Lemma 10, we have

$$
\sum T_{f_{j}} T_{f_{j}}^{*}=\sum T_{g_{j}} T_{g_{j}}^{*} .
$$

Now define operators $F$ and $G$ on $\sum \oplus H^{2}$ by

$$
F_{i j}= \begin{cases}T_{f_{j}} & \text { if } i=1, \\ 0 & \text { otherwise, }\end{cases}
$$

and similarly for $G_{i j}$. The equation above says that $F F^{*}=G G^{*}$. Now, applying Theorem 8 we see that there is a partial isometry $V$, commuting with $S_{\mathbb{C}^{N}}$, so that $V F^{*}=G^{*}$ and $F^{*}=V^{*} G^{*}$. By the remarks preceding Theorem 8, we can think of $V$ as having the form $U \otimes I_{H^{2}}$, where $U$ is a partial isometry acting on $\mathbb{C}^{N}$. It is not hard to see that the partial isometry $U$ is the required operator. 
Actually, the hypothesis in Corollary 11 can be weakened. We do not need a universal bound $C$; the value of the sums need only be bounded on compact subsets of $\Omega$.

Similar results hold for analytic functions on regions in $\mathbb{C}^{n}$, by using Toeplitz operators acting on $H_{C}^{2}\left(D^{n}\right)$, where $D^{n}$ represents the polydisk in $\mathbb{C}^{n}$. There is a new difficulty in this case, namely the verification that the equation $F F^{*}=$ $G G^{*}$ implies that $F \mathcal{E}^{\perp} F^{*}=G \mathcal{E}^{\perp} G^{*}$ for all $\mathcal{E}$ in the lattice of the tensor product, $\bigotimes_{j=1}^{n} T(\mathbb{N})$, where $T(\mathbb{N})$ is the collection of infinite upper triangular matrices. One can handle this problem by writing each such $\mathcal{E}^{\perp}$ as a finite linear combination of products of coordinate shifts and their adjoints. We omit the details.

\section{REFERENCES}

1. W. Arveson, Operator algebras and invariant subspaces, Annals of Math. 100 (3) (1974), 433-532. MR 51:1420]

2. R. G. Douglas, On Majorization, Factorization, and Range Inclusion of Operators on Hilbert Space, Proceedings of American Mathematical Society 17 (1966), 413-415. MR 34:3315]

3. A. Hopenwasser, The Equation $T x=y$ in a Reflexive Operator Algebra, Indiana University Mathematics Journal 29 (1980), 121-126. MR 81c:47014

4. E. Katsoulis, Remarks on the Interpolation and the Similarity Problem for Nest Subalgebras of von Neumann Algebras, Journal of Mathematical Analysis and Applications 190 (1995), 755-762. MR 96b:47051

5. E.C. Lance, Some Properties of Nest Algebras, Proceedings of the London Mathematical Society (3) 19 (1969), 45-68. MR 39:3325

6. H. Meschkowski, Hilbertsche Räume mit Kernfunktion, Springer Verlag, Berlin, 1962. MR 25:4326

7. R.L. Moore and T.T. Trent, Solving Operator Equations in Nest Algebras, Houston Journal of Mathematics 24 (1998), 483-488. MR 2000a:47149

8. R.L. Moore and T.T. Trent, Factoring Positive Operators on Reproducing Kernel Hilbert Spaces, Journal of Integral Equations and Operator Theory 24 (1996), 470-483. MR 97f: 47017

Department of Mathematics, Keimyung University, Taegu, Korea

Department of Mathematics, Taegu University, Taegu 712-714, Korea

Department of Mathematics, Box 870350, University of Alabama, Tuscaloosa, AlABAMA 35487-0350

E-mail address: rmoore@gp.as.ua.edu

Department of Mathematics, Box 870350, University of Alabama, Tuscaloosa, AlABAMA 35487-0350

E-mail address: ttrent@gp.as.ua.edu 